Review

\title{
Microfluidic Electrochemical Detection Techniques of Cancer Biomarkers
}

\author{
Yao Xie, Di Chen ${ }^{-}$Shujing Lin \\ Key Lab for Thin Film and Microfabrication Technology of Ministry of Education, Shanghai Intelligent Diagnosis and Treatment \\ Instrument Engineering Technology Research Center, Department of Instrument Science and Engineering, School of Electronic \\ Information and Electrical Engineering, Shanghai Jiao Tong University, Shanghai 200240, China.
}

Corresponding author. E-mail: dchen@sjtu.edu.cn

Received: Jan. 11, 2017; Accepted: Feb. 28, 2017; Published: Mar. 29, 2017.

Citation: Yao Xie, Di Chen and Shujing Lin, Microfluidic Electrochemical Detection Techniques of Cancer Biomarkers. Nano Biomed. Eng., 20I7, 9(I): 57-7I.

DOI: 10.5101/nbe.v9i1.p57-71.

\begin{abstract}
Microfluidic electrochemical sensors are based on the immune response. Immune charge density or electrode potential and other parameters sensitive interface causes a change or cause a power solution of the active ingredient (or their related components) to generate or consume, causing the detected current or potential. The electrochemical parameters of the law of changing enable the immune proteins quantitative or qualitative detection. Since the immune protein molecules themselves are typically non-electrochemical activity, and therefore immune electrochemical sensor usually mark the electrically active substance or enzyme in the antigen or antibody molecules in solution antigen (antibody) immune response and by the immobilized antigen (antibody) the concentration of the sample antigen (antibody) after the change of the current signal, causing the electro-active substance or enzyme-catalyzed reaction of substrate reaction can be determined indirectly.
\end{abstract}

Keywords: Microfluidic, Cancer biomarkers, Elelctrochemical detection

\section{Introduction}

Cancer is one of the major diseases of serious harm to human health, and the incidence rate showed an increasing trend. Colorectal cancer is one of the top five cancer causing deaths, with the incidence rate showing a clear upward trend. Although its multidisciplinary treatment achieved some progression, metastasis is still the biggest challenge [1-6]. Approximately 30\% of the patients were diagnosed with the presence of colorectal cancer metastases [7], in five years after the primary tumor resection, as well as more than $50 \%$ of patients with liver metastases. Some scholars reported that in the autopsy cases after the deaths of colorectal cancer, liver metastases were up to $36 \%-81 \%$ [8]. Such a high occurrence rate is an important factor affecting the overall level of the treatment of colorectal cancer. Thus, colorectal cancer liver metastases have been a clinical urgency that needs to be addressed as an important issue. So far, patients with metastatic colorectal cancer are still the means of monitoring blood CEA, the lack of early diagnosis of metastasis [9]. In the past five years, the application of targeted drugs has brought patients with colorectal cancer overall two years of unrespectable survival, and the treatment has not been efficient.

Cancer liver metastasis did not occur after a radical surgery and it occurred to most patients sooner or 
later. There is currently no effective way to predict which patients are at high risk of liver metastasis. Postoperative liver metastasis screening high-risk groups, early warning and early intervention are of great significance to improve the prognosis of colorectal cancer. Stomach cancer is the second largest cause of cancer death; it is one of the commonest malignant tumor threat to human health. According to the statistics report released by World Health Organization, world annual incidence rate of gastric cancer was $13.86 / 10$ million, second only to lung cancer. In China, stomach cancer is still the most representative of the high incidence of cancer and mortality [6]. The peak age of onset is 50-60 years, more among males than among females with the ratio of about 2:1. And the past 10 years showed a younger trend. The national annual average population dying of stomach cancer was 22.7 million; the mortality was 23.14/10 million, ranking first in all kinds of cancer mortality. About $80 \%$ of early gastric cancer is asymptomatic, the remaining $20 \%$ does not have specific clinical symptoms, and can easily be considered as peptic ulcers or other gastrointestinal disorders and thus neglected. In clinical practice, early diagnosis rate increased by $1 \%$, about 16 million people in China get a new life, concerned to restore the number to one billion dollars in annual losses. Clinical emphasis should be focused on early diagnosis of gastric cancer. In advanced gastric cancer after surgery, the patient survival rate is only $30-40 \%$ within five years, while in early gastric cancer (EGC) stage, namely infiltration into gastric cancer, the 5-year survival rate after surgery can be $80-90 \%$. And if found in the originating small stage gastric cancer (SGC) and minor stomach cancer (MGC) phase, the ten-year survival rate can be up to $100 \%$ [7]. Therefore, early diagnosis and treatment of gastric cancer have a great significance on reducing the mortality rate of gastric cancer. In China, early gastric cancer detection rate is far lower than the world's advanced level, In Japan, early gastric cancer detection and surgical rate is over $50 \%$; 5 -year survival rate is over $90 \%$. In China, these two figures are less than 5\%. Early diagnosis of gastric cancer is very important in Japan. Barium meal and gastroscopy detection are the most important means of discovering early gastric cancer. However, because these diagnostic instruments strongly rely on technical staff, and are time-consuming and costly, their universal coverage in China is difficult. By contrast, the determination of serum tumor markers has the technical advantages of being simple, lowcost, and easy to promote, it has been widely used in China. Hence, the use of serum tumor markers to detect stomach cancer has become an important area of scientific research. However, how to realize highly sensitive and specific detection of these serum tumor markers is still a very big challenge. How to use new technology to improve the technical methods of the pre-warning and early diagnosis of gastric cancer is a major public health problem in China nowadays.

Bladder cancer is the commonest malignancy of the urinary system, accounting for the fourth in the incidence of cancer among American men, and the sventh among women in the United States in 2012, with 73,510 new cases of bladder cancer, including 14,880 deaths [8]. Chinese Journal of Clinical statistics showed that the incidence of bladder cancer was still in the first place in urinary system tumors, with the male incidence rate of $14.72 / 100,000$ and the female incidence rate of 5.34/100,000 [9]. In the initial diagnosis of all patients, approximately $75 \%$ were nonmuscle invasive bladder cancer (non-muscle-invasive bladder cancer, NMIBC), and 25\% were muscle invasive bladder cancer (muscle-invasive bladder cancer, MIBC) [10]. The five-year survival rate of nonmuscle invasive bladder cancer was greater than $90 \%$, but the recurrence rate was as high as $60 \%-85 \%$, of which $20 \%$ may progress to muscle invasive tumors [11]. According to statistics in the United States, the cost of treatment of bladder cancer is the most costly among all tumors; the average treatment cost per patient is $\$ 202,000$, twice the cost of lung cancer [12]. The latest data showed that in the United States the cost for treatment of bladder cancer was $\$ 3.98$ billion in 2010, which was a heavy economic burden [13] to the community.

Bladder cancer has a rapid and high recurrence rate. To find a noninvasive method for bladder cancer screening in newly diagnosed and postoperative monitoring of high-risk groups, and particularly the search for the diagnosis of bladder cancer with high sensitivity, specificity and convenient monitoring methods have important clinical significance on improving the survival rate, while reducing the cost of inappropriate treatment, and thus have important social significance and economic value.

Early detection and diagnosis is extremely important for cancer prevention and treatment. Studies show that with early detection and timely treatment. $90-95 \%$ of 
cancer can be cured. Tumor marker (tumor marker, $\mathrm{TM}$ ) refers to tumorigenesis and proliferation process, produced by the tumor cell itself or by the body's response to tumor cells produced, and to the presence and growth of tumor response for a class of substances, including proteins, hormones, enzymes, polyamines and oncogene products and so on. Tumor markers in cancer screening, diagnosis, prognosis, evaluation of efficacy and high-risk groups and other follow-up study have great practical value. Tumor marker detection technology is almost the only effective way and means of early detection of asymptomatic. Multiple tumor marker protein chip is a high-throughput, high sensitivity, high-specificity and miniaturized protein analysis technology, but now widely using ELISA kits rely on optical detection method for quantitative detection in clinics. The detection process needs a relatively long time; the operation is relatively complex and costly, unable to achieve multi-channel simultaneous detection of multiple indexes [13, 14].

In 1951, Breyer and Radcliff firstly used polarography method for the determination by the azolabeled antigen, opening the prelude of electrochemical immunoassay. After decades of electrochemical sensors have become immune sensor in the first research [16]. The electrochemical immunosensor refers to biological material as the sensing element, to an antigen or antibody for the molecular recognition element, the electrodes as the conversion element, the sensing element by changes in the immune response of the signal is converted to an electrical signal corresponding to an immunosensor. It combined a variety of different electrical analytical techniques such as chronoamperometry, cyclic voltammetry, stripping voltammetry, pulse voltammetry, and differential pulse method. The electrochemical signal sensors can be divided into several tyoes according to the measurements, including potentiometric immune sensors, current-immune sensors, conductivity-type immune sensors, capacitive sensors and impedance immune sensors. The electrochemical biosensor immune sensor has good selectivity, variety, low cost of testing for the online technology, etc; also the electrochemical detection system may be implemented, from sample color and turbidity effects equipment required equipment is relatively simple, fast and convenient detection of other characteristics. It is widely studied and has been gradually applied in the detection of serum tumor markers.

Electrochemical sensors are based on the immune response. Immune charge density, electrode potential and other parameters sensitive interface can cause a change or a power solution of the active ingredient (or their related components) to generate or consume. Since the immune protein molecules themselves are typically non-electrochemically active, and therefore immune electrochemical sensors usually mark the electrically active substance or enzyme in the antigen or antibody molecules .

\section{Nanomaterial-Based Cancer Markers Electrochemical Detection Technology}

The microscopic structure of nanomaterials has at least one dimension in the nanoscale material within range. Common materials include nano-metal powder materials, non-metallic, inorganic compounds, organic compounds, and others of nanometer range. Due to the small size of nanomaterials many of the conventional materials exhibit significantly different properties, including:

(1) Small size effect refers to the changes of substances with a smaller size caused by optical, electrical, magnetic, thermal, chemical and catalytic activity and other macro-physical properties. Nanomaterials with a small size but a large surface area, will exhibit significant differencies from conventional materials in terms of macroscopic physical properties, such as an increase in the absorption of light and generating plasma resonance absorption peak frequency shift.

(2) Surface effect refers to the nature of the change when the substance is in the nanometer scale. The surface atoms, the specific surface area and surface energy become smaller as the size of increasing. Since the surface atoms are in a bare state, having a high surface energy, they have a high activity and high volatile, and it is easy to combine them with other atoms to form a stable structure.

(3) Quantum size effect: Level spacing of nanomaterials increases with decreasing size, when energy level spacing is greater than energy, electrostatic energy, magnetic energy, photon energy of the superconducting state of aggregation or average energy level spacing when nanomaterials It will exhibit unusual characteristics different from conventional materials. 
(4) Macroscopic quantum tunneling effect: microparticles (whose total energy is less than the potential ridge height) through the barrier arised from changes in macroscopic systems.

Nano-materials have been widely used in electronics, optics, chemistry, machinery, biology and medicine because of their unique nature. In biochemistry analysis, nanomaterials also exhibit a wide range of applications, including biomarkers and imaging, bio-separation and enrichment, super sensitive biological samples analysis, and disease diagnosis.

Recently, immune sensor based on biological enzyme-labeled and combined with the new development of highly sensitive electrochemical nanomaterials has become a popular research point. Séamus et al. used avidin and biotin specific binding [17], prostate cancer antibody (PSA) fixed in the matrix of the sensor, and then using the AC impedance, prepared by the label-free electrical chemoimmunotherapy sensors. For PSA testing, the range of the sensor is $1-100 \mathrm{pg} / \mathrm{mL}$; the detection limit is $5 \mathrm{pg} / \mathrm{mL}$. Electrochemical immunosensor was successfully prepared by fixing the carcinoembryonic antibody (CEA) in a polymer and carbon nanospheres composite top, and then with a CdS-labeled secondary antibody incubation [18]. The CEA detection range of the sensor is $0.032-10 \mathrm{ng} / \mathrm{mL}$; the detection limits of $32 \mathrm{pg} / \mathrm{mL}$. CA125 antibody immobilized on a modified polypyrrole nanowires of silicon substrate was prepared by the conductivity type of immune sensor [19]. The CA125 detection range of the sensor is $1-1000$ $\mathrm{U} / \mathrm{mL}$; the detection limit is $1 \mathrm{U} / \mathrm{mL}$. Warsinke using gold nanoparticles modified electrodes fixed PSA antibody, antigen and after modified with nano-beads crosslinked secondary antibody incubation catalase labeled the sensor detection limit of $0.5 \mathrm{pg} / \mathrm{mL}$ [20]. He using functional nano-carbon spheres immobilized antibody, and nano carbon ball to mark the catalase coupled secondary antibody was prepared by a double signal amplification AFP immune sensor [21]. The linear range was $0.05-6 \mathrm{ng} / \mathrm{mL}$; the detection limit is $0.02 \mathrm{ng} / \mathrm{mL}$. This method has high sensitivity and good reproducibility. Li et al. doped with sulfur gold magnetic nanoparticles which combined with catalase enhancers using for the second antibody labeled [22]. To achieve the ultra-sensitive detection of CEA, the detection range is $0.01-160 \mathrm{ng} / \mathrm{mL}$; the detection limit is $5 \mathrm{pg} / \mathrm{mL}$.

\section{Overvievw of Microfluidic Tech- nology}

Microfluidic chip is a technology platform aimed at replacing the various functions of conventional chemical or biological laboratories, which involved reaction, separation, detection and cell culture, sorting, cracking and other basic operating unit integrated or basic integrated into a few square centimeters on the chip in chemistry and biology, and other fields [23, 24] (Fig. 1). In 1995, HP (later Agilent) and Caliper jointly launched the first commercially microfluidic chip analytical instruments. In the late 1990s, there was a group of scientists involved in this field. The "largescale integrated microfluidic chip" was published in the Science journal which described the integrated

From materials to devices to systems
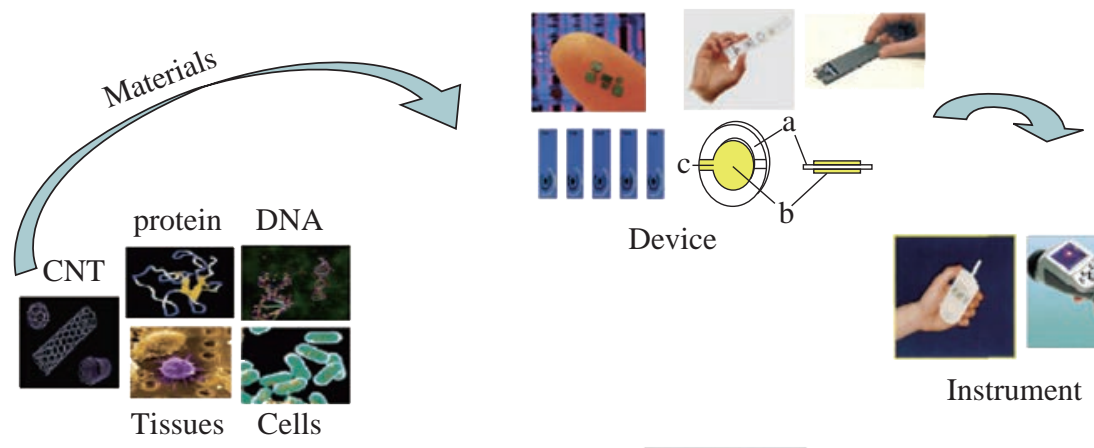

Device

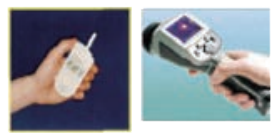

Instrument
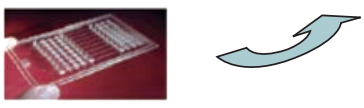

Microfluidics

Fig. 1 Construction of a cancer marker sensor element contained in microfluidics [15]. 
valves and hundreds of reactor chip, showed a simple electrophoresis large-scale multi-functional integrated laboratory in the chip [25]. Today microfluidic chip technology has been listed as one of the most important cutting-edge technology of the 21 st century. October 2003, in the special issue of commemoration of its 85th anniversary, Forbes magazine listed the 15 most important inventions of human influence on the future; Microfluidic chip was one of them. As we can see, a variety of cell technology of microfluidic chip has a flexible combination of controllable and integration features. It presents a lot of single cell technology features unmatched in the research process, especially its integrity and systematic. A microfluidic system has made great achievements in many areas. Since the microfluidic system can greatly reduce the analysis timeand the risk of contamination. with the system have low power consumption, high reliability and sensitivity, also it can be integrated multi-channel analysis, especially in the individual to take care of possible applications. The basic characteristics and the biggest advantage of microfluidic lab cell technology are the more flexible combination of the miniature scale integration. Compared with conventional experiments, microfluidic chip has many unique advantages:

(1) Less sample and reagent: microfluidic chip size determines the amount of reagent can reach nanoliter or picoliter, which greatly reduces the consumption and analysis costs of reagent, especially that of valuable biological reagents, and thus reduces the cost of experiments.

(2) Short analysis time and high efficiency: microfluidic reaction is usually in nanoliter and can be automatically measured in tens of seconds of time; microfluidic reaction also can be a complete test of the different components, can achieve highpass volume applications, which can improve test efficiency.

(3) High sensitivity and accurate results: The amount of various reagents in the microfluidic reactor can be precisely controlled; the reaction conditions can be accurately controlled, which can further improve the accuracy of the reaction, to obtain more accurate results. It is possible to accurately analyze the results produced by different components.

(4) Miniaturization and portability: microfluidic chip technology will be combined flexibly in a variety of units on the tiny platform and largescale integration of its basic characteristics, which is also its greatest advantage. So you can use microfluidic chip production fully functional portable instrument for all types of on-site analysis.

According to the existing published papers and patents, the development trend of micro flow control chip has the following main aspects:

(1) Instrument miniaturization and miniaturization: study of microfluidic systems, mainly in capillary electrophoresis on chip miniaturization, miniaturization of the detector research is still inadequate. In order to achieve the goal of portability, further miniaturized and integrated detection system is imperative. In terms of miniaturization and integration testing equipment, the primary task is to achieve breakthrough in fluorescence and electrochemical detectors. In recent years, the semiconductor laser volume reduction, the emergence of short-wavelength diode lasers, the light-emitting diode have provided favorable conditions for fluorescence detector miniaturization. Development of micro-processing technology for integrated electrochemical detector provides an effective technology platform.

(2) System integration: microfluidic chip analysis systems has been in development for more than a decade, and its increasingly gaining high degree of integration. But so far the main innovations are more concentrated on the separation and detection system. The study on how to respond to actual sample analysis of the many issues on the chip, such as the introduction of the sample before treatment is still very weak.

(3) The size of the microfluidic chip integrated: One of the important advantages of microfluidic analysis system by MEMS technology is its ability to form a certain scale of integration. With the current general level of MEMS technology, processing hundreds of micron-sized channels or other features of the microstructure on a chip with only a few square centimeters is not difficult. The multiple parallel microfluidic is implemented to significantly improve efficiency and reduce any possible cost of analysis.

(4) The development of a variety of detection techniques: the variety of micro total analysis system, initially induced fluorescence detection 
method, until now which has remained fairly widespread application. The more and more of a semiconductor laser have gradually been used in some applications, but more for absorption spectrophotometry detection [22]. With the improvement of micro-processing capacity, electrochemical detection method has achieved rapid development in recent years [23].

Microfluidic chip is used as an analytical device, which is one of the main areas of application in biomedical and clinical diagnostics, such as genotyping, DNA sequencing, cell research and other fields. This review studies the use of microfluidic chip in electrochemical detection and screening of serum blood cells. Serum is obtained through the microfluidic chip filter and on-chip integrated electrochemical detection devices, and then the information is read from the electrical signal in the sample.

\section{Microfluidic-Based Electroche- mical Detection Technology}

Analytical methods in accordance with the carrier and the immune response can be divided into homogeneous electrochemical immunoassay analysis and heterogeneous immunoassays. Homogeneous immunoassay utilizing liquid phase antigenantibody reaction is detected. Then the heterogeneous immunoassay antibody or antigen is immobilized on a solid carrier. In recent years, based on electrochemical and homogeneous-phase immunoassay, microfluidic chips have made great progress. The former principal use of biological molecules electrophoresis separation on a microchip for analysis, while the latter is the higher sensitive than the use microchannels surface area to improve the performance of immunoassay methods.

In terms of quantitative methods, electrochemical immunoassay microfluidic chips can be divided into non-competitive immunoassays and competitive immunoassays. In competitive immunoassays, analyte and labeled antigen compete for binding to the active site on the limited antibody. The lower the concentration of labeled antigen is bound to a solid support, the lower the signal is, when the detection signal is antigen-antibody complexes.. In the amount of antibody can obtain the maximum sensitivity, the sensitivity depends on affinity of the antibody. The detection signal intensity is proportional to the concentration of the antigen. Diffusion process has a great impact on the strength of the detection signal [2427].

In terms of detector means, immune microfluidic chip electrophoresis analysis can be divided into optical detection and electrochemical detection, of which the commonest is the fluorescence detector. Many of the devices are based on electrophoresis using laser induced fluorescence detection, because it has good sensitivity and short response time. In terms of fluorescence detection method, the microscope is usually focused on the microchannel, and then connected with the CCD reading optical signal. The other optical detection method further comprises a chemiluminescent [28-29] and heat through a microscope $[30,31]$ and so on. Electrochemical detection methods are commonly used, including amperometric, potentiometric method, and capacitance method. The use of current voltammetry curves and fixed voltages can make amperometric immunoassay. When combined with microfluidic chip, electrochemical detection can be divided into flow-through, the flow type and flow-through [32]. Advantages of microfluidic chips voltammetry are applied to detecting tiny electrodes without reducing the immunoassay results. In this paper, the first classification is reviewed.

\section{Heterogeneous Microfluidic Elec- trochemical Detection of Cancer Markers}

Heterogeneous immuneoassay requires separation of free and bound label prior to detection. Microfluidic chip-phase immunoassay mostly antibodies (sometimes antigen) immobilized on a solid support. The advantage is in the antigen from dilute solutions which is concentrated to a solid phase carrier surface enriched by washing, you can achieve the antigen-antibody complex and separation of free antigen or antibody. This method is characterized with small antibody loss, rapid analysis, can be reused, high sensitivity and low detection limits. The different antibodies are fixed to different parts of the microchip, form an array, and thus achieve polyhydric immunoassay.

\section{The use of beads as the solid support}

Microfluidic immunoassays use beads as a solid support which is a unique advantage compared to conventional immunoassays. Firstly, the detection 
areas for microchip increase. For example, $0.1 \mu \mathrm{m}$ in diameter of beads can produce about $60 \mathrm{~m}^{2}$ of surface area [13]. Antigen or antibody is immobilized on the surface of the microbeads, and can effectively reduce the diffusion of sample to the surface, thus reducing the incubation time of the immune response, and accelerating the immune response analysis rate. Secondly, the use of physical fields including pressure field, electric field and magnetic field can effect surface modification antigen or antibody beads to transfer into micro ducts. Thirdly, the beads may have different surface modification methods, introducing different functional groups to play different roles in the microfluidic chip. Finally, the magnetic field can be controlled in the microchannels. Ko et al [32] developed a detecting three cancer markers (AFP, CEA, PSA) of the microfluidic device.
Based on the sandwich form, polystyrene beads are connected by biological avidin antibody. The catalytic solution is formed on the surface of metallic silver nanoparticles, then after deposition, increases the gold particle diameter, and thus plays the role of a bridge constructed in a platinum microelectrode bottom, and thereby reduces the resistance, and thus play effect detected (Fig. 2).

Electrochemical immunoassays are used to some of the more common antibody - antigen electrochemical test. Sato developed a micro quartz weir structure, the adsorbed immunoglobulin A (s-IgA) chip fill with polystyrene microbeads, microbeads entrapped in micro weir at the glue binding gold flowing s-IgA reaction channels with beads $[15,16]$. They also used this chip and beads to develope a sandwich immunoassay method (Fig. 3), and with the same

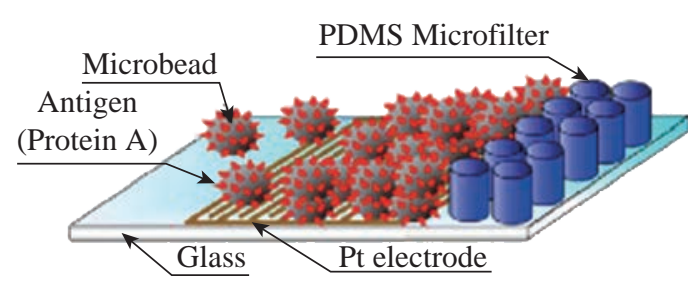

(a)

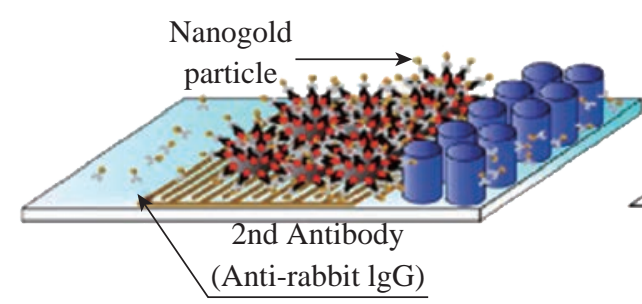

(c)

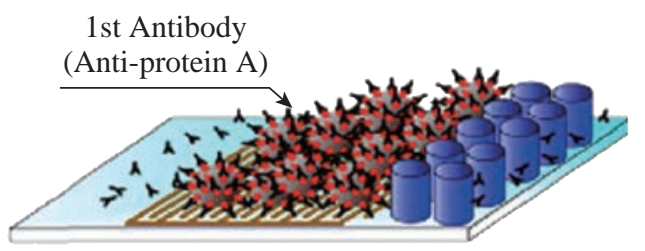

(b)

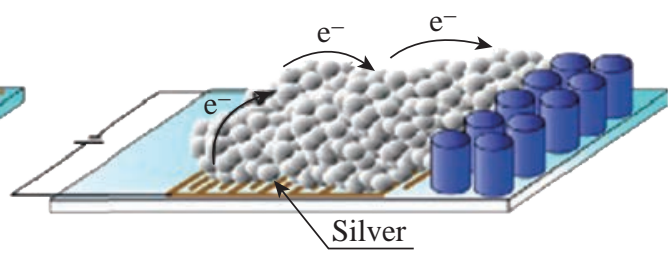

(d)

Fig. 2 Electrical analysis and detection: (a) surface covered with antigen bead enrichment; (b) the antigen-antibody reaction; (c) an antibody-gold coupling reaction; (d) silver beads wrapped amplified signal [32].

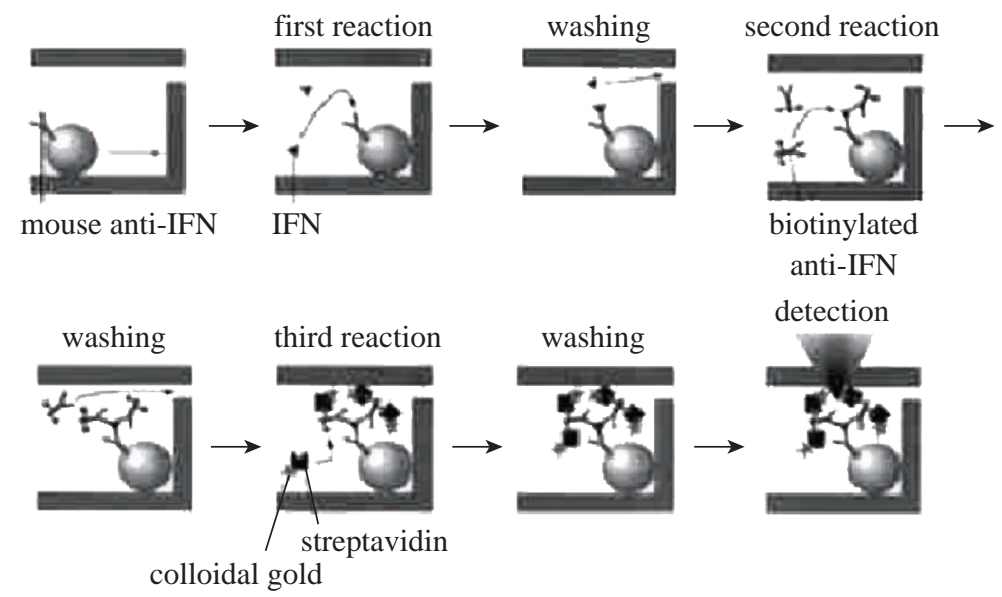

Fig. 3 Immunosorbent microfluidic chip schematic analysis [16]. 
principle further proposed a four-channel microfluidic chip immunoassay (Fig. 4), so that the analysis efficiency is greatly improved.

Using polystyrene beads as a carrier has been extensively studied in combination immunoassay fluorescence detection. Haes et al fabricated a microfluidic chip pipe [32] (Fig. 5) with a double weir structure (double-weir structure), using fixed microbeads. In the surface of the polystyrene beads modified by chemical methods staphylococcal enterotoxin B (SEB) antibodies, and is fixed to the channel. The fluorescent labeled antigen flow through the pipe and sufficiently reacted with beads, and then SEB was added unlabeled antigen. Fluorescence unlabeled antigen avidity and affinity of the antibody is much greater than the labeled antigen and antibody. Labeled antigen will be replaced at outlet, and unlabeled antigen and antibody immune complex is formed, antigen signal on the microarray. Proportional to the signal strength and antigen concentration, analysis takes only $20 \mathrm{~min}$. The detection limit was $28.5 \mathrm{fg} / \mathrm{ml}$ ); the linear range spanning six orders of magnitude (1 fM-1 nM). The system for detecting the actual biological samples (bovine serum albumin, casein and milk) in the target biomolecules also has a high sensitivity $(10 \mathrm{pM})$, but the actual biological sample pretreatment is required prior to testing. In addition, the optimization of the experimental program of the washing step can improve the quantitative nature of the process. The sample will be contributed to the (a)

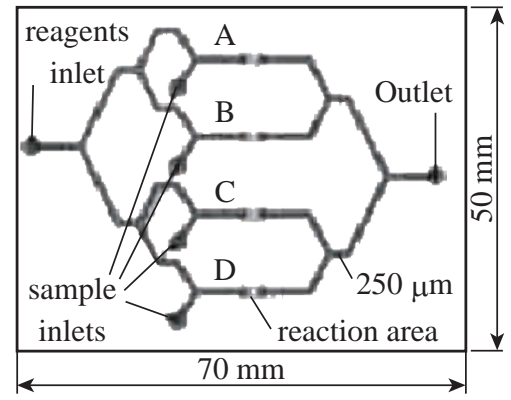

(b)

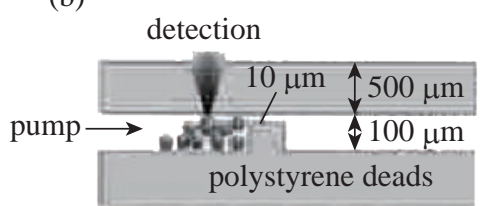

(c)

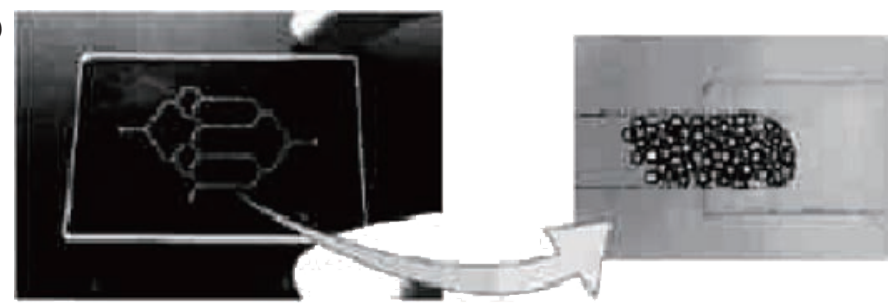

Fig. 4 Immunosorbent assay quartz glass microchip: (a) a schematic view; (b) reaction and the detection site; (c) microchips and microbeads gather photograph [17].

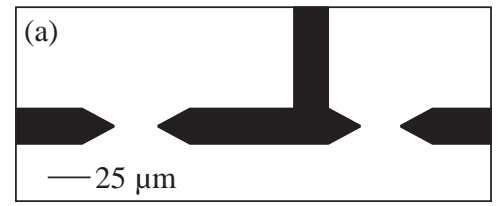

(c) 1

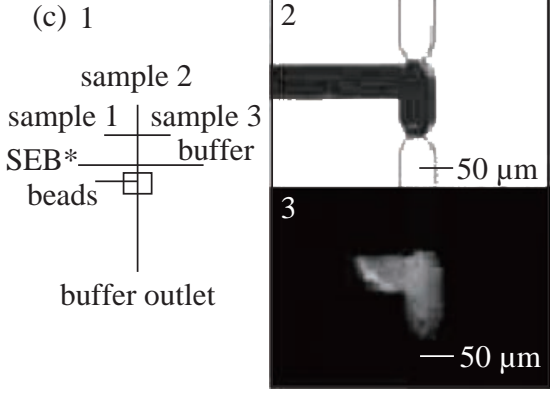

(b)

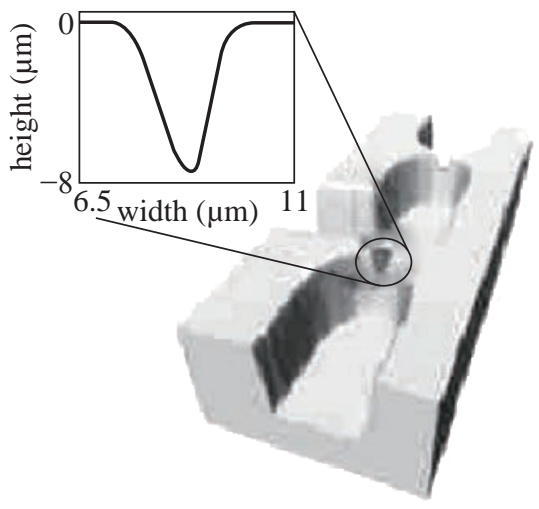

Fig. 5 The microchip with dual weir structure [32]. 
development of new methods of microfluidic sample pre-enrichment.

Shin et al made a microbeads cavity microfluidic chip [33]. In the first, they used off-chip chemically modified antibodies on the microbeads, the antigen and the antigen fluorescently labeled and then hit by a negative pressure into the chamber, and the fluorescence reaction of detection were happened in the chamber. Yasukawa et al using of negative dielectrophoresis techniques formed a cage in the microchannel [34], in which the sandwich immunoassay and fluorescence detection took place. The reaction time was $40 \mathrm{~min}$; the detection limit was $1.4 \mathrm{nM}$. The magnetic beads can be used as solid support. This can be controlled with a magnet on the beads. Hayes et al using beads as a fixed vector [35], the pressure-driven flow established on-chip immunoassay system. Microfluidic chip does not require special processing structure; the magnetic beads is fixed to the channel surface, performed immunoassay sandwich method, the immune response of timewas only $10 \mathrm{~min}$. Fluorescence detection of the reaction measured discharge microbeads at the end of the retreat to the magnetic field. This approach simplifies the microfluidic chip processing with a more convenient operation. Choi et al developed a electromagnet embedded microfluidic chip channel [36], the antibody-modified magnetic beads introduced to microchannels, the different antigens flow through the channel. It occurs only with the target antigenspecific response. After that the reaction is incubated with the antigen immobilized on magnetic beads labeled. Finally the magnetic field can achieve the purpose of measuring target antigens from different kinds of antigens. Kim et al developed an outer chip beads fluorescently labeled by the sandwich method which was connected to an antibody, the antigen and labeled super paramagnetic nanoparticles [37]. Since the magnetic field is perpendicular to the channel, the magnetic and non-magnetic beads are separated during flow todetecte with a fluorescence detector. The microfluidic chip used to rabbit IgG and mouse IgG simultaneous detection, detection limits reached 244 $\mathrm{pg} / \mathrm{ml}$ and $15.6 \mathrm{ng} / \mathrm{ml}$. However, the immune response are all outside the chip; it takes multi steps and is timeconsuming ( $30 \mathrm{~min}$ or more). The chip only does the separation and the detection, but is not conducive to on-site testing [38-39]. Dodge also used as a carrier immune response microbeads sandwich method. All reactions were outside the chip [40]. Microbeads were injected into the chip after the reaction. The use of enzyme-catalyzed substrate was subjected to electrochemical detection. After repeated use of fixed antibodies, the immune reactivity may decrease. In order to ensure the accuracy of the results and the reproducibility, each of bead recyclable are use to surfaceso that each of analysis is used the new antibody modified microbeads [41-42]. Compared to other fixed carrier antibodies, antibody immobilized carrier beads ares easier to achieve surface renewal, but filled with beads and exhaust operates with a certain degree of difficulty. Beads may also be adsorbed on the channel walls or electrode, blocking the channel and reducing channel inner or scatter light.

\section{The use of channel wall for support}

Antibody immobilized on the surface in the channel has a high specific surface area. There are direct fixation adsorption and covalent bonding on the wall. Hydrophobic interaction can be fixed directly to the wall of the antibody, but because of its conformation changes, the reactivity is decreased. In order to reduce the protein molecules and small molecules in the non-specific adsorption [21]. According to Liu et al consisting of PMMA carried polyimide (PEI) modified itself surface, the modification of surface usingamine functional groupscan be combined with the antibody [39]. Covalently linked with glutaraldehyde as liver cancer markers AFP, in the introduction of two top anti-horseradish peroxidase HRP sandwich structure constituted by using a secondary antibody labeled AFP concentration, and carry out differential pulse volt in $\mathrm{H}_{2} \mathrm{O}_{2}$ and 2-amino-phenol An assay (DPV), the detection limit can reach $1 \mathrm{pg} / \mathrm{mL}$. According to Dodge et al channels in the local area glass chip by physical adsorption of protein $\mathrm{A}$. The use of protein $\mathrm{A}$ and rabbit immunoglobulin (rIgG) allows higher affinity between the antibody and the antibody on the channel walls [40]. This method avoids direct modification of the antibody in the channel walls of its active site which cannot be fully exposed to the outside, and r-IgG immunoassay using direct and competitive immunoassay. Eteshola et al. reported that the PDMS chip achieved a heterogeneous immunoassay sheep immunoglobulin IgM [41]: first, the BSA were modified in a microchannel wallusing the glutaraldehyde-activated, and then bonded protein A to the BSA of inner wall, thereby enabling the capture of antibodies specifically bonded to protein A. When sheep IgM analyte through the microchannel was captured by primary antibody labeled with horseradish peroxidase (HRP) secondary 

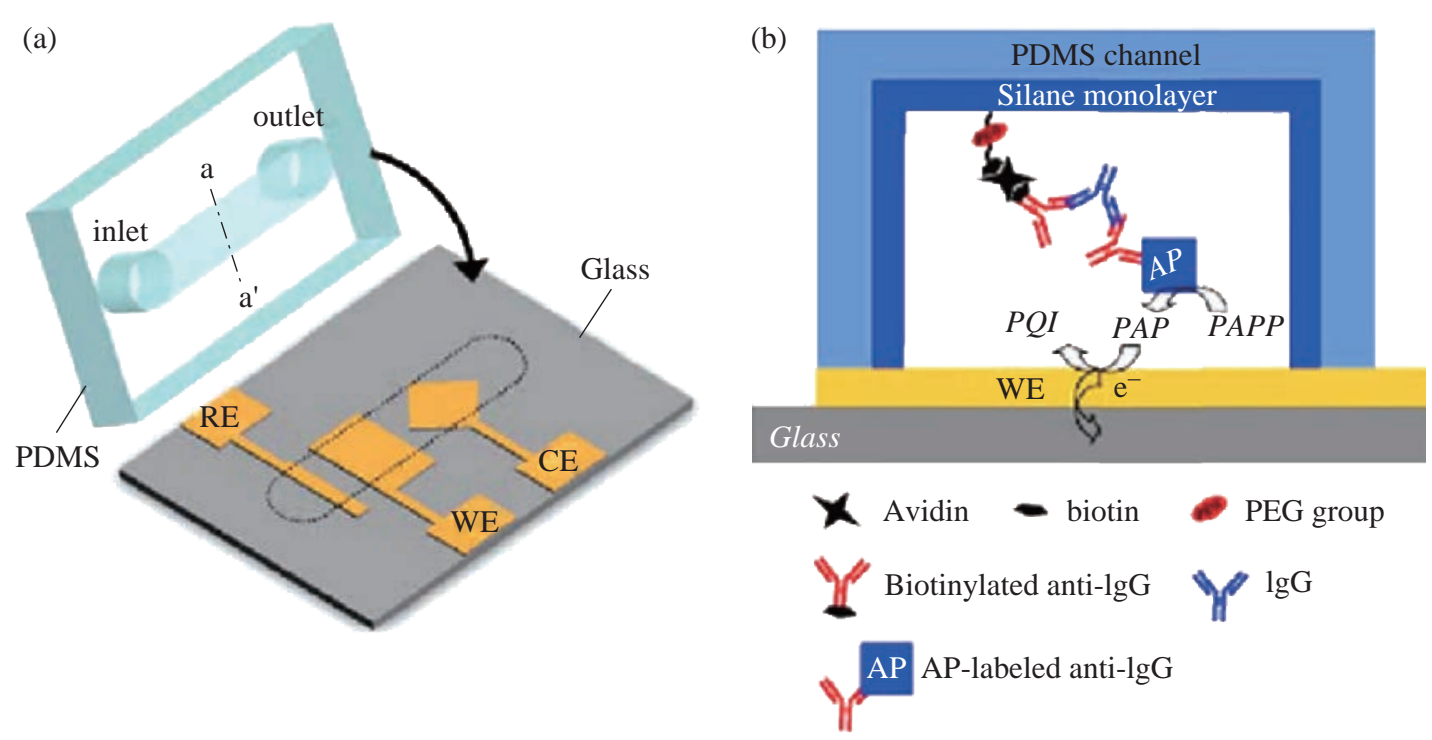

Fig. 6 (a) Utilizing the assembly of three electrodes immune PDMS chip. WE: working electrode; CE: counter electrode; RE: reference electrode; (b) inside the PDMS pipes immunity measurement [44].

antibodies catalytic substrate p-hydroxyphenyl pyruvic acid (HPPA) oxidized to produce fluorescent substance immunoassay, the detection limit was $17 \mathrm{nmol} / \mathrm{L}$. Jang et al reported a three-electrode system in the machining surface of the glass substrate and the surface of integrated surface functionalized PDMS channel [42]. In the PEG-modified PDMS channel surface monolayer and connected biological avidin, PEG reduces the effect of non-specific adsorption caused by biological avidin which is used to connect antibody [43, 44, 45] (Fig. 6). Their group also developed IDA electrode based microfluidic chips which is also used for immunoassays.

Hosokawa developed a unpowered injection immunoassay such as the use of air high solubility in PDMS and diffusion speed characteristics. The chip structure is simple and easy to use [45] (Fig. 7). The sandwich-phase immunoassays have been studied. The first antibody modifications to the inner wall, then using fluorescence assay rabbit $\operatorname{IgG}$ and human $\mathrm{C}$-reactive protein (C-reactive protein). The immunoassay time (including antibody fixed time) was $20 \mathrm{~min}$; the detection limits were $0.21 \mathrm{nmol} / \mathrm{L}$ and 0.42 $\mathrm{nmol} / \mathrm{L}$. According to Rossier et al., carbon electrodes were embedded in the channel wall, and then the antibody were fixed in the channel surface [46]. Since the microchannel has a large surface area and small diffusion distance immunological reagents, each step of the immune response incubation takes only $5 \mathrm{~min}$.

\section{The electrode surface as a solid support}

Some microfluidic immunoassay chips are only for micro-channel flow control, and the immune reaction take place the electrode surface and is detected. Aguilar et al. fabricated the microelectrodes integrated microfluidic immunoassay chip, effectively shortening the analysis time, saving the immunological reagents, and achieving a better sensitivity [47]. Dong et al. produced a double annular electrodes embedded (dualring electrodes) immunoassay microchip [48]. On this basis, they turn around the electrode placed in a cavity embedded with a similar electrodes structure. The use of micro-channel reagents introduced into the chamber for analysis, As shown in Fig. 8 [50]. The chip integrates the entire analysis steps; including a probe which is fixed, sample injection, incubation and electrochemical detection. Sandwich immunoassay
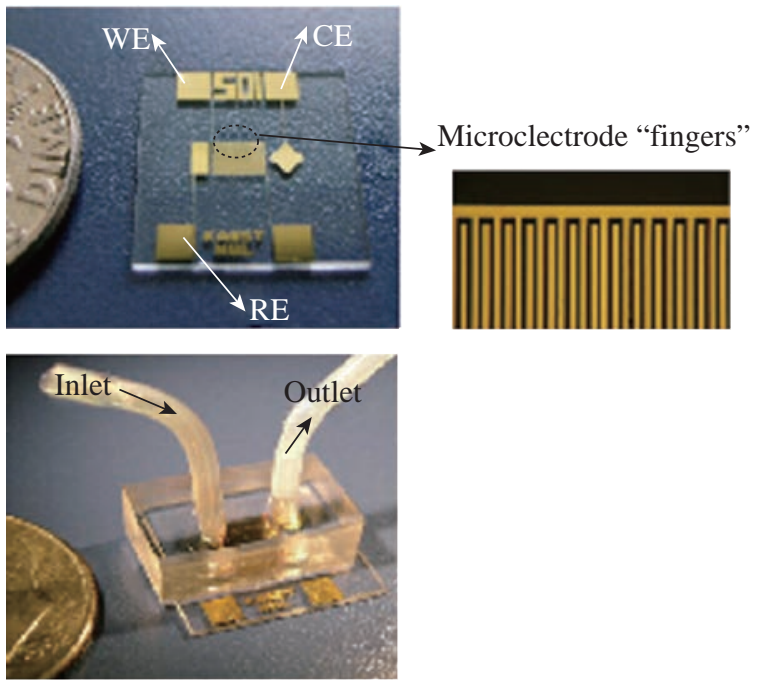

Fig. 7 Photos of IDA gold electrode chip [45]. 


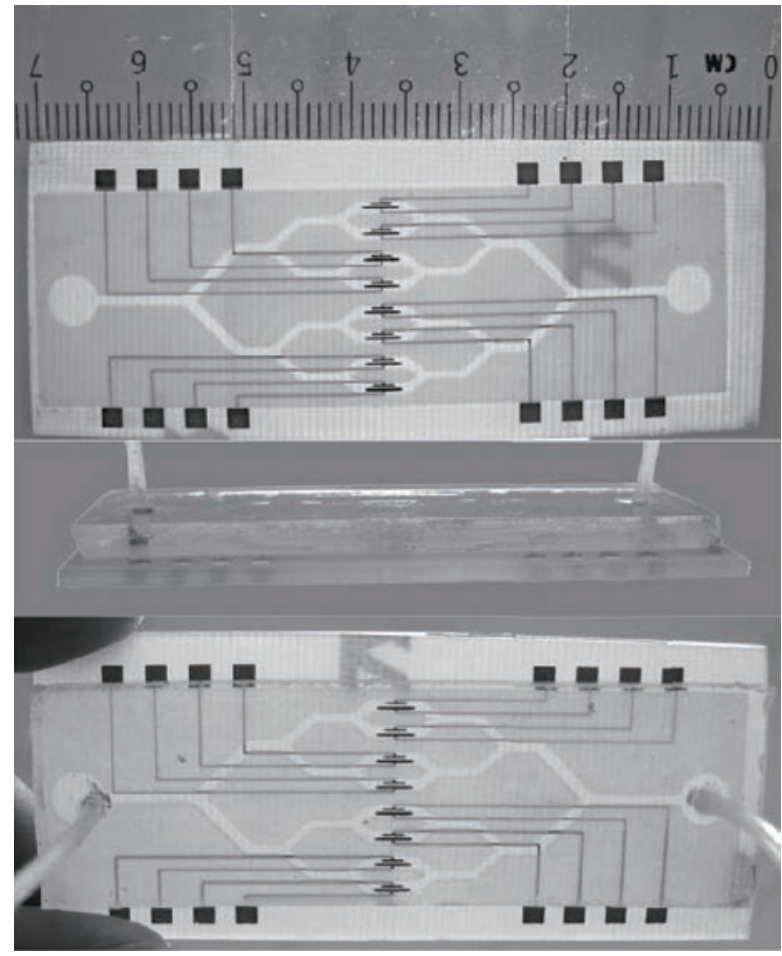

Fig. 8 A schematic diagram of the microfluidic chip electrochemical immunoassay [50].

was performed for the mouse IgG; the detection limit was $10 \mathrm{ng} / \mathrm{ml}$, the linear of range was in the range of $100 \mathrm{ng} / \mathrm{ml}-10 \mu \mathrm{g} / \mathrm{ml}$. Nashida et al. developed a valve controlling injection and washing microfluidic chip [50]. Micro-channel liquid in the central pipeline will be sandwich immune response and electrochemical detection. Such microchips in common are that the modified antibody electrodes are enclosed in a chamber. The use of electrochemical detection signal enzymatic reaction in a substrate enables a high sensitivity and selectivity. During the analysis, automated fluid control device simplifies the steps to improve the efficiency of the analysis. The chip production process is relatively complicated, including micro-valves, channels, the embedded electrode modification and the like. As onetime use, the cost is large, but for repeated use, the modified electrode replacement operation will be more complicated. However, some improvement in the chip design can achieve multi-channel immunoassays.

\section{Homogeneous Microfluidic Electrochemical Detection Technique of Cancer Markers}

Homogeneous microfluidic cancer marker immunological reagents and analytes technique was present to design some structures on the microfluidic chip in the same phase, so that they flow into the pool from a different immune response in the micromicro-channel. Since immunological reagents and substrates used the liquid phase, the flow operation is relatively easy, and it is easy to achieve a variety of analysis functions integrated. After a homogeneous immunoassay antibody is immobilized and washing steps, without regard to the solid phase non-specific adsorption on the carrier, but the immune reaction formed a composite which normally took the mixture to separate in order to achieve detection.

Homogeneous electrochemical immunoassay antibody-antigen complex generates a flag signal. No fractional binding and reactions in the immune process, which produces a number of heterogeneous electrochemical immunoassay, because of no antibody were immobilized and washed steps, there is no solid non-specific adsorption. But homogeneous reaction and the detection limit is often worse than the sensitivity heterogeneous reaction. Homogeneous reaction
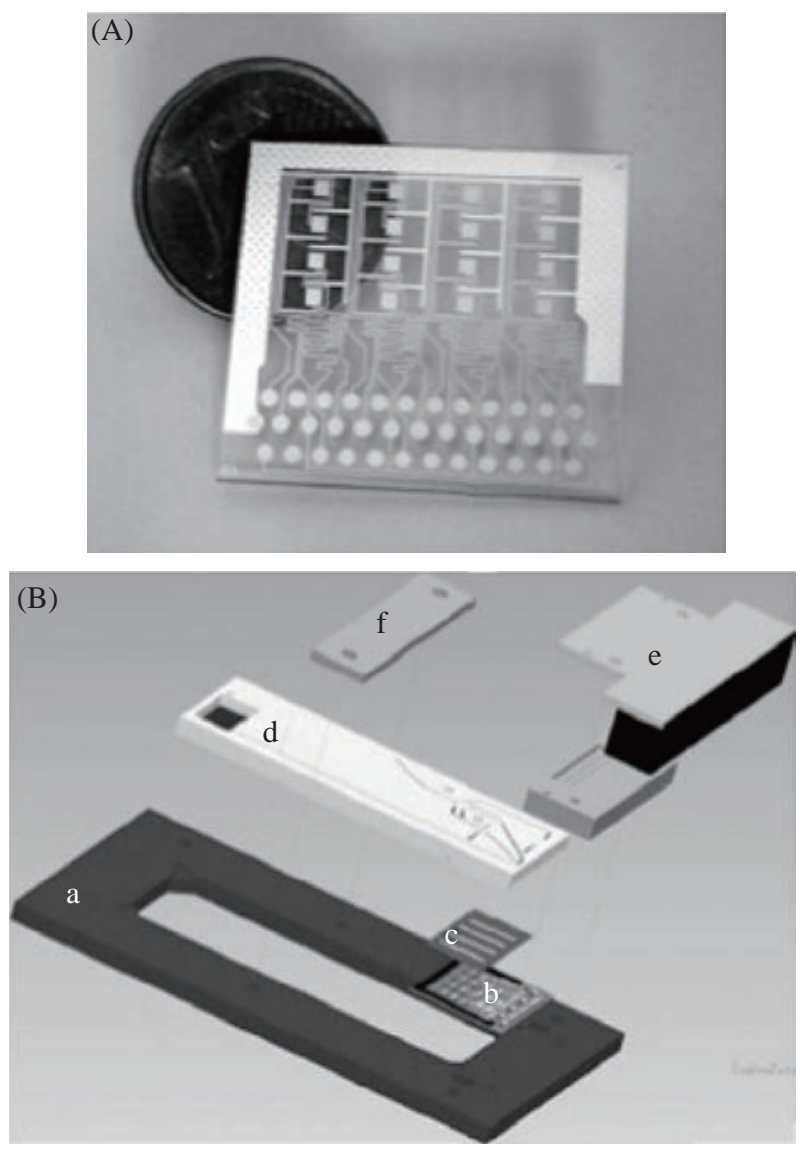

Fig. 9 (A) 16 square silver gold electrode and the common electrode and silver reference electrode electrochemical immunoassay array; (B) microfluidic chip electrode array Annex: (a) holder; (b) the electrode array; (c) two-sided duct tape adhesion; (d) fluid chip; (e) plug connector; (f) strut [52]. 
(a) Interdigitated type electrode

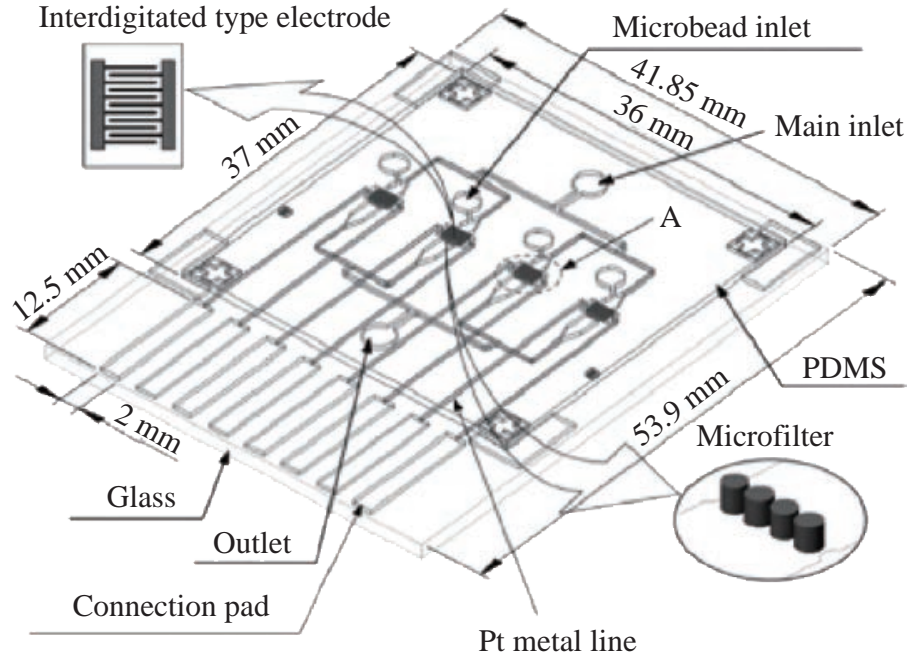

(b)

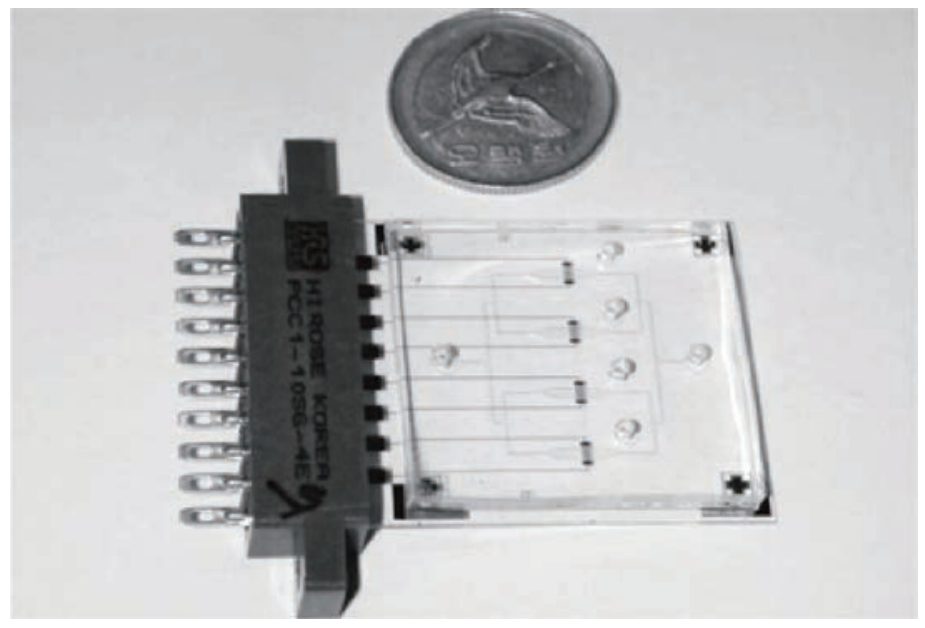

Fig. 10 PDMS-glass electrochemical immunoassay sensor chip: (a) 3D design; (b) chip photo [53].

usually requires higher detection concentration, and the purification step requires special samples. The most commonly application is electrochemical capillary electrophoresis (CE), and antibody immune complexes based on their different electric mobilities are distinguished. Zhang et al reported that the CEA and AFP of biomarkers were labeled antibodies; the immune complexes were isolated and injected into the sample cell, and then injected the sample cell in another enzyme complex; electrochemistry is performed at the end of the pipeline amperometric detection chamber. The detection time of the four immune complexes (anti-AFP-HRP, anti-CEA-HEP, AFP-anti-AFP-HRP and CEA-anti-CEA-AFP) were in less than $60 \mathrm{~s}$ [51].

\section{Multi-Biomark Detection Tech- nology}

Multi-samples analyzed using a single microfluidic chip is becoming a trend, especially in biology and clinical diagnosis. The sample includes a variety of analytes. Henry et al. invented a low-density electrode array chip for detecting cancer markers (Fig. 9). 16 electrodes are arranged in the form of $4 \times 4$ in the middle of the "sandwich" type of chip, wherein the chip includes a fluid conduit, a sump, valves, and the detection electrode. 16 detection electrodes can handle the signals generated by CEA and PSA [52].

Ko et al. designed a microfluidic chip as shown in Fig. 10 [53]. The chip was fabricated in the electrodes on the glass as well as PDMS piping components. An entry is added to the sample and reagents; another entrance to combine with an antigen beads and another outlet. Micro-pillars at each monitoring chamber capture microbeads. This method can simultaneously detect CEA, AFP and PSA markers.

Chen et.al developed an amicrofluidic chip including blood cell filtration, antigen capture, online testing. Preliminary work has been carried out blood cells in 


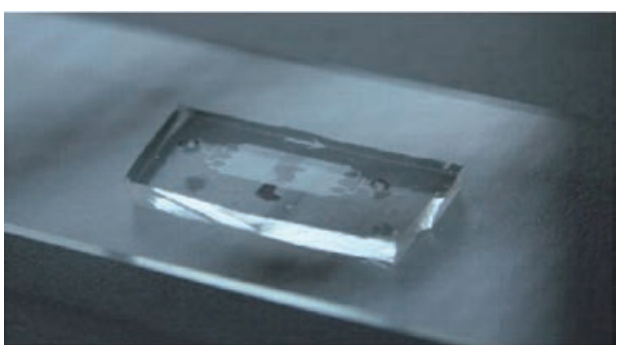

(a)

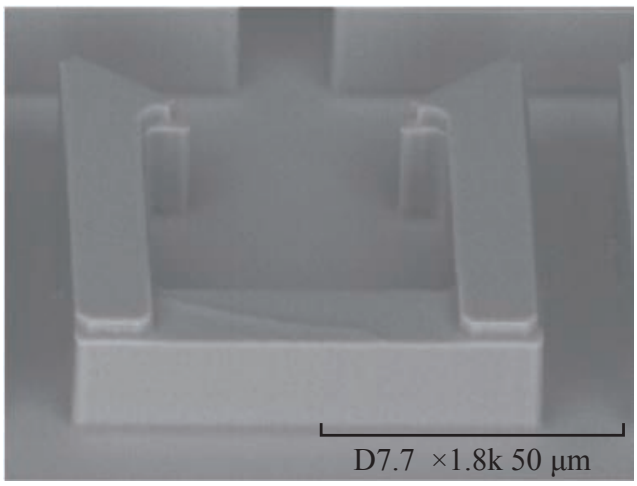

(d)

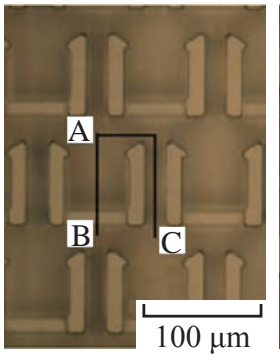

(b)

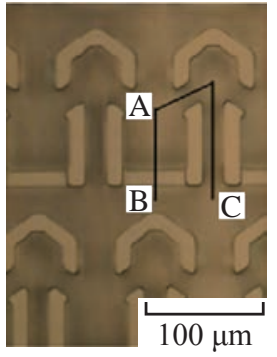

(c)
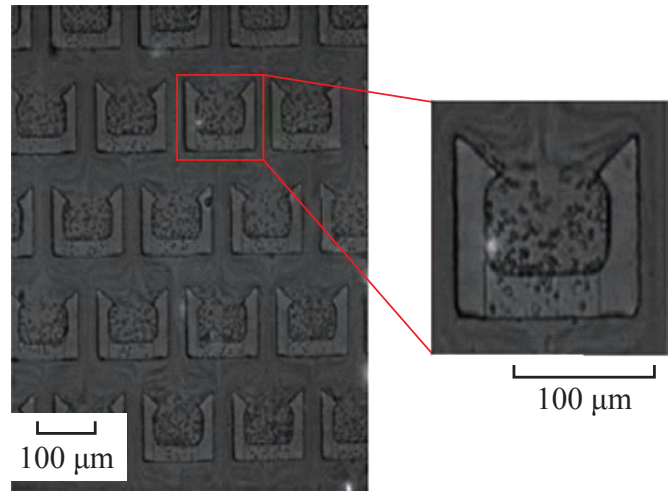

(e)

Fig. 11 (a) Blood cell filter chips; (b) without diversion cap structure; (c) there are diversion cap structure; (d) without diversion cap structure SEM photographs; (e) photographs capture blood cells [54, 55].

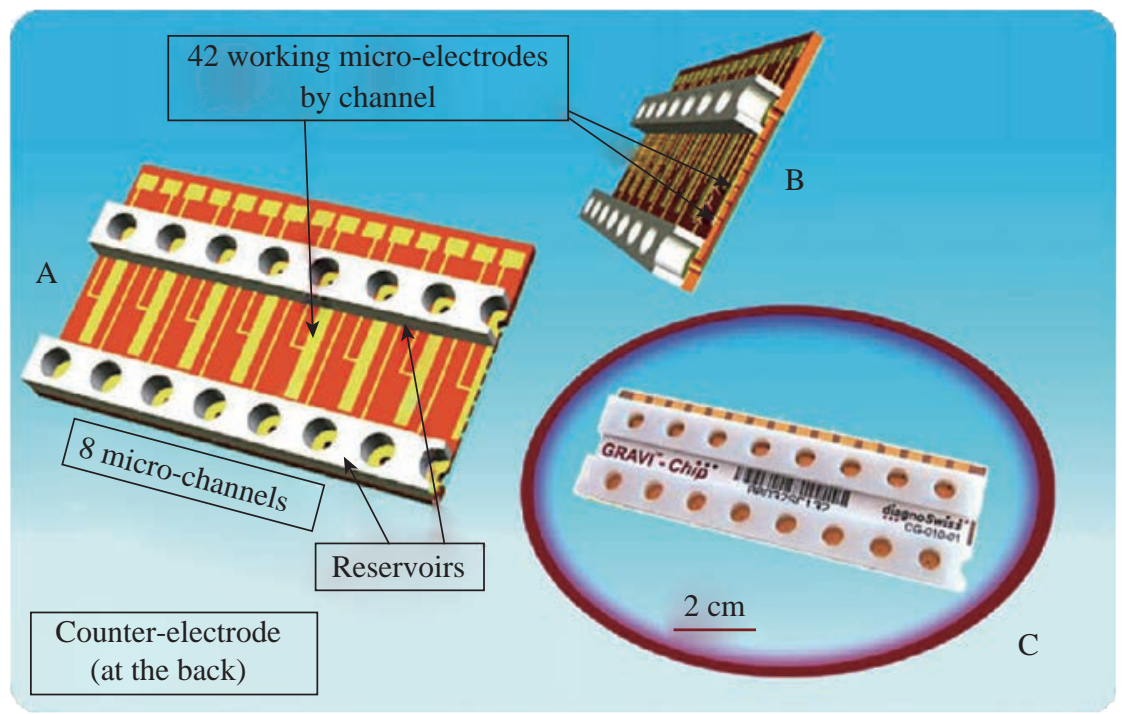

Fig. 12 GRAVI-Chip: (a) the upper 8-channel chip, reservoirs and circuit, a cross-sectional view; (b) micro-pipe and integrated electrode array; (c) the commercialization of GRAVI-Chip [56].

micro-structure of all filters (Fig. 11) for immunoassays detection of ongoing serum $[54,55]$.

Reymond [56] reported the development of an automated microfluidic immunoassay platform. The immune chip consists of a set of gold electrodes which are arranged linearly, independent and separate from each of components in channel. Similar to the traditional 96 -well plates, the micro-pipe is in accordance with the automation to match with the other devices. Each immune chip can run eight independent experiments (Fig. 12).

\section{Conclusions}

Electrochemical detection of cancer markers in multiple interdisciplinary technologies is the result 
of comprehensive development. Electrochemical sensing technology makes cancer immune markers more sensitive and effective. The microfluidic system integrates markers' capture, detection and reading on one chip, greatly reducing the testing cost and improving the detection efficiency. The new technology enables the integration of multiple on-chip laboratories which have become more powerful. The future trend in electrochemical detection of cancer marker technology is toward a smaller, portable, high-throughput system with a large range of applications from detecting a single cancer to predicting the further development of the various stages of prognosis of cancer.

\section{Acknowledgments}

This work was supported by the Key Research and Development Plan of Ministry of Science and Technology (No. 2016YFC0100902), the Science and Technology Commission of Shanghai Municipality (No. 15441904800) and the Shanghai Economy and Informatization Commission (No. XC-ZXSJ-02-2016$05)$.

\section{References}

[1] N.Y. Shih, H.L. Lai, G.C. Chang, et al., Anti- $\alpha$-enolase autoantibodies are down-regulated in advanced cancer patients. Aticle title. Jpn J Clin Oncol., 2010, 40: 663669.

[2] T. Szarvas, M. Becker, F. Vom Dorp, et al., Matrix metalloproteinase-7 as a marker of metastasis and predictor of poor survival in bladder cancer. Cancer Sci., 2010, 101: 1300-1308.

[3] I.E, Tothill, Biosensors for cancer markers diagnosis. Seminars in Cell \& Developmental Biology, 2009, 20: 5562.

[4] B. Breyer, F.J. Radcliff. Polarographic investigation of the antigen antibody reaction. Nature, 1951, 167: 79-79.

[5] C.B. Andrew, D. Frank, P.J. Séamus, Labeless immunosensor assay for prostate specific antigen with pictogram per milliliter limits of detection based upon an ac impedance protocol. Anal. Chem, 2008, 80: 6198-6205.

[6] D.X. Cui, L. Zhang, X.J. Yan, et al., A microarraybased gastric carcinoma prewarning system. World $J$ Gastroenterol, 2005, 11(9): 1273-1282.

[7] D.X. Cui, et al., Present situation and development prospects of nano-tumor diagnosis and treatment technologies. Chinese Journal of Cancer Biotherapy, 2008, 15(5): 401-406.

[8] R. Siegel, D. Naishadham, Cancer statistics, CA Cancer J Clin., 2012, 62(1): 10-29.

[9] W. Zhang, Y.B. Xiang, Z.W. Liu, et al., Trends analysis of common urologic neoplasm incidence of elderly people in Shangha. Cancer, 2004, 23(5): 555-558.

[10] M. Babjuk, M. Burger, R. Zigeuner, et al., EAU guidelines on non-muscle-invasive urothelial carcinoma of the bladder: update 2013. Eur Urol. 2013, 64(4): 639653.
[11] R.F. Youssef, Y. Lotan, Predictors of outcome of nonmuscle-invasive and muscle-invasive bladder cancer. Scientific World Journal, 2011, 11: 369-381.

[12] K.D. Sievert, B. Amend, U. Nagele, et al., Economic aspects of bladder cancer: what are the benefits and costs? World J Urol., 2009, 27(3): 295-300.

[13] A.B. Mariotto, K.R. Yabroff, Y. Shao, et al., Projections of the cost of cancer care in the United States: 2010-2020. J Natl Cancer Inst., 2011, 103(2): 117-128.

[14] J.A. Ho, Y.C. Lin. L.S. Wang, et al., Carbon nanoparticleenhanced immunoelectrochemical detection for protein tumor marker with cadmium sulfide biotracers. Anal. Chem. 2009, 81: 1340-1346.

[15] M.A. Bangar, D.J. Shirale, W. Chen, et al., Single conducting polymer nanowire chemiresistive lable-free immunosensor for cancer biomarker. Anal. Chem., 2009, 81: 2168-2175.

[16] A. Warsinke, A. Benkert, F.W. Scheller, et al., Electrochemical immunoassays. Anal. Chem., 2000, 366: 622-634.

[17] G.F. Jie, J.J. Zhang, D.C. Wang, et al., Surface plasmon resonance study of protein-carbohydrate interactions using biotinylated sialosides. Anal. Chem., 2008, 80(11): 4033-4039.

[18] D. Tang, R. Yuan, and Y. Chai, Ultrasensitive electrochemical immunosensor for clinical immunoassay using thionine-doped magnetic gold nanospheres as labels and horseradish peroxidase as enhancer. Anal. Chem., 2008, 80: 1582-1588.

[19] B.C. Lin, Microfluidic chip laboratory and functional. China Pharmaceutical University, 2003, 34(1): 1-6.

[20] B.C. Lin, J.H. Qin, Microfluidic lab on a chip. Chromatography, 2005, 23(5): 456-463.

[21] T. Thorsen, S.J. Maerkl, and S.R. Quake, Microfluidic large-scale integration. Science, 2002, 298(5593): 580584.

[22] C.T. Lim, Y. Zhang, Beas-based microfluidic immunoassays: The next generation. Biosens. Bioelectron, 2007, 22: 1197-1204.

[23] A. Bange, B.H. Halsall, W.R. Heineman, Microfluidic immunosensor systems. Biosens. Bioelectron., 2005, 20: 2488-2503.

[24] A. Bhattacharyya, C.M. Klapperich, Design and testing of a disposable microfluidic chemiluminescent immunoassay for disease biomarkers in human serum samples. Biomed. Microdevices, 2007, 9: 245-251.

[25] K. Sato, M. Tokeshi, T. Odake, et al., Integration of an immunosorbent assay system : analysis of secretory human immunoglobilin A on polystyrene beads in a microchip. Anal. Chem., 2000, 72: 1144-1147.

[26] K. Sato, M. Tokeshi, H. Kimura, et al., Determination of carcinoembryonic antigen in human sera by integrated bead bed immunoassay in a microchip for cancer diagnosis. Anal. Chem., 2001, 73: 1213-1218.

[27] K. Sato, M. Yamanaka, H. Takahashi, et al., Microchipbased immunoassay system with branching multichannels for simultaneous determination of interferon- $\gamma$. Electrophoresis., 2002, 23: 734-739.

[28] W.R. Vandaveer, S.A. Pasas-Farmer, D.J. Fischer, et al., Recent developments in electrochemical detection for microchip capillary electrophoresis. Electrophoresis., 2004, 25: 3528-3549.

[29] L.B. Koutny, D. Schmalzing, T.A. Taylor, et al., Microchip electrophpretic immunoassay for serun cortisol. Anal. Chem., 1996, 68: 18-22.

[30] N. Chiem, D.J. Harrison, Microchip-based capillary electrophoresis for immunoassays : analysis of monoclonal antibodies and thephylline. Anal. Chem., 1997, 69: 373-378.

[31] C. Leng, X.Q. Zhang, H.X. Ju. Microfluidic chip-based 
immunoassay. Prog Chem., 2009, 21: 688-695.

[32] A.J. Haes, A. Terray, G.E. Collins, Bead-assisted displacement immunoassay for staphylococcal enterotxin B on a microchip. Anal. Chem., 2006, 78: 8412-8420.

[33] K.S. Shin, S.W. Lee, K.C. Han, et al., Amplification of fluorescence with packed beads to enhance the sensitivity of miniaturized detection in microfluidic chip. Biosens. Bioelectron., 2007, 22: 2261-2267.

[34] T. Yasukawa, M. Suzuki, T. Sekiya, et al., Amplification of fluorescence with packed beads to enhance the sensitivity of miniaturized detection in microfluidic chip. Biosens. Bioelectron., 2007, 22: 2730-2736.

[35] M.A. Hayes, N.A. Polson, A.N. Phayre, et al., Flow-based microimmunoassay. Anal. Chem., 2001, 73: 5896-5902.

[36] J.W. Choi, C.H. Ahn, S. Bhansali, et al., A new magnetic bead-based, filterless bio-separator with planar electromagnet surfces for integrated bio-detection systems. Sens. Actuators B., 2000, 68: 34-39.

[37] K.S. Kim, J.K. Park, Magnetic force-based multiplexed immunoassay using superparamagnetic nanoparicles in microfluidic channel. Lab Chip, 2005, 5: 657-664.

[38] S. Farrell, N.J.R. Matsuno, H.B. Halsall, et al., Beadbased immunoassays with microelectrode detection. Anal. Bioanal. Chem., 2004, 379: 358-367.

[39] Y. Liu, H. Wang, J. Huang, et al., Analysis of bioactive ingredients in the brown alga fucus vesiculosus by capillary electrophoresis and neutron activation analysis. Anal. Chim. Acta., 2009, 650: 77-82.

[40] A. Dodge, K. Fluri, E. Verpoorte, et al., Electrokinetically driven microfluidic chips with surface-modified chambers for heterogeneous immunoassays. Anal.Chem., 2001, 73: 3400-3409.

[41] E. Eteshola, D. Leckband, Development and characterization of an ELISA assay in PDMS microfluidic channels. Sens.Actuators B, 2001, 72: 129-133.

[42] Y. Jang, S.Y. Oh, J.K. Park. In situ electrochemical enzyme immunoassay on a microchip with surfacefunctionalized poly(dimethylsiloxane) chanel. Enzyme Microb. Technol., 2006, 39: 1122-1128.

[43] C. Yang, C. Zhao, L. Wold, et al., Biocompatibility of a physiological pressure sensor. Biosens. Bioelectron. 2003, 19: 51-58.

[44] C.B. Cohen, E.C. Dixon, S. Jeong, et al., A microchipbased enzyme assay for protein kinase A. Anal. Biochem., 1999, 273: 89-97.

[45] K. Hosokawa, M. Omata, K. Sato, et al., Power-free sequential injection for microchip immunoassay toward point-of care testing. Lab Chip., 2006, 6: 236-241.

[46] J.S. Rossier, H.H. Girault, Enzyme linked immunosorbent assay on a microchip with electrochemical detection. Lab Chip, 2001, 1: 153-157.

[47] Z.P. Aguilar, W.R. Vandaveer, I. Fritsch, Enzyme linked immunosorbent assay on a microchip with electrochemical detection. Anal. Chem., 2002, 74: 3321-3329.

[48] H. Dong, C.M. Li, Q. Zhou, et al., Sensitive electrochemical enzyme immunoassay microdeviced based on architecture of dual ring electrodes with sensing cavity chamber. Biosens. Bioelectron., 2006, 22: 621-626.

[49] H. Dong, C.M. Li, Y.F. Zhang, et al., Screen-printed microfluidic device for electrochemical immunoassay. Lab Chip., 2007, 7: 1752-1758.

[50] N. Nashida, W. Satoh, J. Fukuda, et al., Electrochemical immunoassay on a microfluidic device with sequential injection and flushing function. Biosens. Bioelectron., 2007, 22: 3167-3173.

[51] S. Zhang, W.J. Cao, M. Li. MCE enzyme immunoassay for carcinoembryonic antigen and alpha-fetoprotein using electrochemical detection. Electrophoresis., 2009, 30: 3427.

[52] O.Y. Henry, A. Fragoso, V. Beni, et al., Design and testing of a packaged microfluidic cell for the multiplexed electrochemical detection of cancer markers. Electrophoresis., 2009, 30: 3398-3403.

[53] Y.J. Ko, J.H. Maeng, Y. Ahn, et al., Microchip-based multiplex electro-immunosensing system for the detection of cancer biomarkers. Electrophoresis., 2008, 29: 34663476.

[54] J.D. Chen, D. Chen, Y. Xie, et al., Microfluidic chips for cells capture using 3-D hydrodynamic structure array. Microsyst Technol., 2014, 20: 485-491.

[55] J. D. Chen, D. Chen, Y. Xie, et al., A microfluidic chip for rapid and direct trapping white blood cells from whole blood. Biomicrofluidics., 2013, 7: 034106.

[56] J.S. Rossier, S. Baranek, P. Morier, et al., GRAVI-chip : Automation of microfluidics affinity assay based on magnetic nanoparticle. J. Assoc. Lab. Automat., 2008, 13: 322 .

Copyright $\Subset 2017$ Yao Xie, Di Chen, and Shujing Lin. This is an open-access article distributed under the terms of the Creative Commons Attribution License, which permits unrestricted use, distribution, and reproduction in any medium, provided the original author and source are credited. 